\title{
Miocardiopatia periparto em gestante jovem do terceiro trimestre: um relato de caso com desfecho favorável
}

\author{
Peripartum cardiomyopathy in a young pregnant woman in the third trimester: a case \\ report with favorable outcome
}
Miocardiopatía periparto en una joven embarazada en el tercer trimestre: reporte de un caso con evolución favorable

Nathalia Pestana Malheiro ${ }^{*}$, Vitória de Barros Carvalho¹, Leonora Alves Ribeiro¹, Eduardo Migani Teixeira².

\begin{abstract}
RESUMO
Objetivo: Relatar um caso de Miocardiopatia Periparto em paciente jovem e previamente hígida para atentar os profissionais ao diagnóstico precoce desta patologia ainda muito subdiagnosticada. Detalhamento de caso: Gestante BFS, 23 anos, G3P1A1C1, previamente hígida, com idade gestacional de 37 semanas, admitida no Pronto Socorro da Santa Casa de Franca com queixa de mal estar e sensação de pré-sincope, com relatos prévios de dispneia progressiva, chegando à apresentar dispneia de repouso e ortopneia. Evoluiu com quadro de insuficiência cardíaca (fração de ejeção < $29 \%$ ), choque cardiogênico, edema agudo de pulmão e complicações fetais como a bradicardia fetal. Após estabilização, foi realizado a resolução da gestação, tratamento adequado e acompanhamento cardiológico no puerpério, recuperando a função do ventrículo esquerdo alguns meses após o quadro. Considerações finais: Apesar da patologia ser rara, devido sua alta morbimortalidade, deve ser lembrada como diagnóstico diferencial em gestante com sintomas como dispneia, comum também em uma evolução normal de gestação.
\end{abstract}

Palavras-chave: Gestante, Cardiomiopatia periparto, Insuficiência cardíaca.

\begin{abstract}
Objective: To report a case of Peripartum Cardiomyopathy in a young and previously healthy patient to assist professionals in early diagnosis of this pathology, still very underdiagnosed. Detail of the case: Pregnant woman BFS, 23 years old, G3P1A1C1, previously healthy, with a gestational age of 37 weeks, was admitted to the Emergency Room of Santa Casa de Franca with a complaint of malaise and a feeling of pre-syncope, with previous reports of progressive dyspnea, even presenting dyspnea at rest and orthopnea. She developed heart failure (ejection fraction <29\%), cardiogenic shock, acute lung edema and fetal complications such as fetal bradycardia. After stabilization, resolution of pregnancy, adequate treatment and cardiological follow-up in the puerperium was performed, recovering left ventricular function a few months after the condition. Final considerations: Although the pathology is rare, due to its high morbidity and mortality, it must be remembered as a differential diagnosis in pregnant women with symptoms such as dyspnea, also common in a normal evolution of pregnancy.
\end{abstract}

Keywords: Pregnant, Peripartum cardiomyopathy, Heart failure.

\section{RESUMEN}

Objetivo: Comunicar un caso de Miocardiopatía Periparto en un paciente joven y previamente sano para ayudar a los profesionales en el diagnóstico precoz de esta patología, aún muy infradiagnosticada. Detalle del caso: Embarazada BFS, 23 años, G3P1A1C1, previamente sana, con edad gestacional de 37 semanas, ingresada en Urgencias de Santa Casa de Franca con consulta de malestar y sensación de presíncope, con antecedentes reportes de disnea progresiva, incluso presentando disnea en reposo y ortopnea. La paciente evolucionó con insuficiencia cardíaca (fracción de eyección <29\%), shock cardiogénico, edema agudo de pulmón y complicaciones fetales como bradicardia fetal. Luego de la estabilización, se realizó la resolución del embarazo, el tratamiento adecuado y el seguimiento cardiológico en el puerperio, recuperando la función del ventrículo izquierdo a los pocos meses de la afección. Consideraciones finales: Aunque la patología es rara, por su alta morbimortalidad, debe recordarse como diagnóstico diferencial en gestantes con síntomas como la disnea, que también es común en una evolución normal del embarazo.

Palabras clave: Gestante, Cardiomiopatía periparto, Insuficiencia cardiaca.

\footnotetext{
${ }^{1}$ Universidade de Franca (UNIFRAN), Franca - SP. *E-mail: mailto:vih.barros94@gmail.com

${ }^{2}$ Fundação Santa Casa de Misericórdia de Franca, Franca - SP.
} 


\section{INTRODUÇÃO}

A miocardiopatia é definida como doença do miocárdio associada a disfunção cardíaca. Quando associadas ao final da gestação ou principalmente ao puerpério, são chamadas de miocardiopatia periparto (MCP). É uma condição rara, incidindo em 1/3000-4000 partos e sua etiologia é desconhecida mas parece estar relacionada à interação fisiológica da gestação e do puerpério com fatores inflamatórios, infecciosos, genéticos, hormonais e metabólicos (SIMÕES M, et al., 2015; MOIOLI M, et al., 2010; SLIWA K, et al., 2010; PYATT J e DUBEY G, 2011).

Em relação aos fatores de risco estão relacionados a idade materna avançada, multiparidade, melanodermia, gestação múltipla, pré-eclampsia e hipertensão gestacional, alto consumo de sódio e baixo nível sócio-econômico. A mortalidade pode chegar a 50\% tendo como principais causas o tromboembolismo, insuficiência cardíaca (IC) grave e arritmias. $E$ entre os fatores prognósticos para uma boa evolução do caso estão o diagnóstico e conduta precoces (AVILA W, et al., 2002; RACHED H, et al., 1998; ROMBALDI A, et al., 2005; DEMAKIS J e RAHIMTOOLA S, 1971).

Os sinais e sintomas são de uma IC que se iniciam no último trimestre ou primeiros 6 meses pós-parto, com dispneia, dispneia paroxística noturna, tosse e hemoptise. Outros ainda podem se confundir aos da gestação normal como astenia, desconforto respiratório e abdominal. Deve-se afastar doença cardíaca prévia, abuso de drogas, diabetes gestacional, síndromes hipertensivas da gestação, tireotoxicose, endocardite e edema pulmonar não cardiogênico (DEMAKIS J e RAHIMTOOLA S, 1971; PATTA C e NUNES R, 2012; LEONARD R, et al., 1992).

Os critérios para diagnóstico de MCP são: Desenvolvimento de IC congestiva no último mês de gestação ou até 5 meses após o parto, ausência de outra causa identificável para a IC, ausência de cardiopatia até 1 mês antes da gestação e disfunção ventricular esquerda pelos critérios ecocardiográficos clássicos, como fração de encurtamento ou fração de ejeção (FE) diminuída (< 45\%) (OLIVEIRA FM, et al., 2012; TEDOLDI C, 2009; PINTO C, et al., 2007).

Durante a investigação diagnóstica o traçado do eletrocardiograma (ECG) pode incluir taquicardia sinusal, fibrilação atrial, anormalidades inespecíficas do segmento ST-T, baixa voltagem, hipertrofia ventricular esquerda. Ondas Q podem estar presentes. Os intervalos PR e QT podem estar prolongados (HOMANS DC, 1985; RESENDE B, et al., 2009).

Para auxiliar na elucidação diagnostica a radiografia de tórax pode demonstrar sinais de cardiomegalia, com aumento do índice cardio-toracico (ICT), edema intersticial, derrame pleural e congestão pulmonar. O ecocardiograma (ECO) bidimensional demonstra aumento ventricular esquerdo com diminuição da função ventricular com ausência de hipertrofia, aumento do átrio esquerdo, regurgitação mitral e tricúspide e derrame pericárdico pequeno. A cineangiocoronariografia pode ser executada se a paciente apresenta fatores de risco para doença arterial coronariana, a qual pode ser realizada no pré-parto (OLIVEIRA FM, et al., 2012; HOMANS DC, 1985; CÈNAC A, et al., 1992).

O tratamento é o mesmo da IC, no qual abrange medidas não farmacológicas como a mudança de estilo de vida, incluindo hábitos alimentares saudáveis e restrição de sal. Entre as medidas farmacológicas está a administração de betabloqueadores, digoxina e outros agentes inotrópicos, dopamina e dobutamina e diurético (ROMBALDI A, et al., 2005; RESENDE B, et al., 2009; PEARSON G, et al., 2000; RICKENBACHER P, et al., 1994).

Ainda, o inibidor da enzima conversora de angiotensina (IECA) faz parte dos medicamentos modificadores da doença, os quais inibem remodelamento cardíaco, é contraindicado nas gestantes, pois é prejudicial ao feto. Porém, após o parto será a medicação mais importante. O uso de anticoagulantes está indicado diante de disfunção ventricular esquerda grave, trombo no ventrículo esquerdo, ou história de embolização. Quando o tratamento medicamentoso é ineficiente, o balão intra-aórtico e o transplante cardíaco são realizados como última opção (ROMBALDI A, et al., 2005; RESENDE B, et al., 2009; PEARSON G, et al., 2000; RICKENBACHER P, et al., 1994). 
Apesar dessa patologia ser rara, sua importância se dá na alta taxa de morbimortalidade, causados pela IC, tais como fenômenos tromboembólicos, arritmias, choque séptico e insuficiência hepática e falência múltipla de órgãos. O planejamento familiar é fundamental, porque as pacientes que normalizaram a função ventricular podem apresentar recidiva da doença em gestação subseqüentes (PATTA C e NUNES R, 2012; RESENDE B, et al., 2009).

O objetivo principal deste relato é atentar os profissionais de saúde quanto à gravidade, os desfechos desfavoráveis e a importância do diagnóstico precoce na condução de gestantes e puérperas com sinais e sintomas cardiorrespiratórios, diferenciando-os de uma gestação fisiológica.

\section{DETALHAMENTO DE CASO}

$O$ relato a seguir foi consentido pela paciente para ser publicado, sendo assinado um Termo de Consentimento Livre Esclarecido (TCLE), garantindo que seu caso tem o objetivo de colaborar com fins científicos e que não será exposto sua identificação ou qualquer dado pessoal.

Gestante B. F. S, 23 anos, G3P1A1C1, do lar, branca, previamente hígida, em acompanhamento de prénatal ambulatorial, com idade gestacional (IG) de 37 semanas pela data da última menstruação, foi admitida no Pronto Atendimento da Santa Casa de Franca-SP (SCF) com queixa de dor tipo contrações esporádicas, associadas a mal-estar e sensação de pré sincope. Relatou ainda que há 1 mês iniciou dispneia de esforço progressivo, evoluindo até dispneia de repouso e ortopneia, associado a leve precordialgia. Referia boa movimentação fetal e negava perda de liquido e sangramento. De antecedentes pessoais relatou história de Epilepsia em uso de Lamotrigina $50 \mathrm{mg}$ 8/8h. Solicitado cardiotocografia que mostrou feto ativo, prescrito sintomáticos e deixada em observação por 1 hora.

$\mathrm{Na}$ reavaliação a paciente queixava-se de dormências e suspeitando de uma aura epiléptica, foi administrado Fenobarbital EV e enquanto corria a medicação paciente evoluiu com piora do quadro, acompanhada de hipotensão grave (pressão arterial (PA) $50 \times 30 \mathrm{mmHg}$ ), desconforto respiratório com queda da saturação de oxigênio e sensação de sufocamento, seguida de sincope. Foi iniciado expansão volêmica, sem resposta, a PA tornou-se inaudível, sendo administrado Noradrenalina EV lentamente, e com ausência de resposta, foi optado por iniciar anti-histamínico suspeitando de Anafilaxia e em alguns minutos houve melhora da PA, porém a gestante apresentou taquicardia supraventricular sustentada, sendo medicada com Adenosina, revertendo a taquicardia.

Após esse evento, paciente apresentou Bradicardia Fetal, sendo encaminhada ao Centro Obstétrico, consciente e orientada, com queixa de precordialgia após as medicações. Realizado ECG que mostrou taquicardia sinusal, eixo à direita, infra desnivelamento de segmento ST em V3,V4,V5,V6. Manteve hipotensão e precordialgia, sendo realizado reposição hídrica com melhora da vitalidade fetal.

Devido à gravidade do caso, no dia seguinte, paciente foi transferida para Hospital do Coração, em unidade coronariana, encontrando-se dispneica, em franco edema agudo de pulmão e hipotensa. Foi iniciado ventilação não invasiva com $2 \mathrm{~L} \mathrm{O} 2$ em cateter nasal, diurético e drogas vasoativas, com melhora da instabilidade hemodinâmica e da dispneia. Realizado ECO a beira do leito, evidenciando redução da fração de ejeção $(\mathrm{FE})<29 \%$, hipocinésia difusa de ventrículo esquerdo (VE) de grau importante, insuficiência miocárdica de grau discreto.

Ao exame físico apresentava bulhas arrítmicas, hiperfoneticas com presença de terceira bulha, sem sopros. Respiração espontânea com frequência respiratória (FR) 20 irpm, SatO2 96\%, murmúrio vesicular presente bilateralmente e reduzido bibasal, ausência de crepitações. Além disso, apresentava picos de hiperglicemia. Ao exame obstétrico havia boa movimentação fetal e batimentos cardíacos fetais dentro da normalidade, sem perdas vaginais.

Após estabilização, paciente retornou a SCF sendo programada a resolução da gestação para o dia seguinte, devido à gravidade do caso e boa vitalidade fetal. Procedimento sem intercorrências, sendo realizado parto cesáreo com IG 37 semanas e 2 dias com laqueadura tubárea por riscos de futuras gestações. 
Após o procedimento puérpera foi conduzida ao Centro de Terapia Intensiva para estabilização, a qual evoluiu bem, realizando desmame gradual das drogas vasoativas. Então, foi solicitado avaliação cardiológica, sendo prescrito Furosemida 40mg, Espironolactona $25 \mathrm{mg}$, Carvedilol 6,25mg, Enalapril $10 \mathrm{mg}$, ajustadas as doses até a alta.

Paciente recebeu alta hospitalar no décimo primeiro dia de internação, assintomática e ECG normal. Devido quadro MCP grave foi encaminhada ao Ambulatório de Cardiologia. Com relação ao seguimento, alguns meses após ao quadro, novo ECO sem alterações, com FE de 52\%.

\section{DISCUSSÃO}

A MCP apresenta como fatores de risco a idade materna avançada, multiparidade, raça negra, gemelaridade, uso de cocaína, beta-agonistas por mais de 4 semanas, história de doença hipertensiva da gestação e antecedente de miocardite. Em contrapartida, este relato descreve uma gestante jovem, sem comorbidades cardiovasculares previas ou vícios, branca e em gestação única, tornando-o atípico (SANTOS SM, et al., 2012).

A doença é um desafio diagnóstico, que acaba sendo muitas vezes tardio ou Subdiagnosticado, pois seus sintomas assemelham-se ao de uma evolução de gestação normal. Entre eles estão edema de membros inferiores, fadiga, dispneia. O diagnóstico precoce é importante para se evitar complicações do quadro, como insuficiência cardíaca, choque cardiogênico, arritmias cardíacas e tromboembolismo. Por se tratar de um diagnóstico difícil, a gestante em questão apresentou uma MCP com as seguintes complicações maternas: Insuficiência cardíaca, choque cardiogênico e a arritmia cardíaca (taquicardia supraventricular diagnosticada pelo ECG). Além disso, o sofrimento fetal, pode estar presente como uma das complicações, que no caso em questão foi demonstrado pela bradicardia ao exame obstétrico (OLIVEIRA FM, et al, 2012; SLIWA F, et al., 2006; DAVIS M, et al., 2019; BORDIGNON S, 2007).

O diagnóstico diferencial é importante, principalmente com IC prévia latente, que pelas alterações hemodinâmicas fisiológicas da gestação, descompensa, ou com tromboembolismo pulmonar, a préeclâmpsia, infarto agudo do miocárdio e miocardite infecciosa. Neste caso, poderia deixar dúvidas quanto ao diagnóstico de uma IC prévia latente, entretanto a mesma apresentou melhora da FE alguns meses após o parto, reforçando a hipótese diagnóstica de MCP (OLIVEIRA FM, et al., 2012; NAPOLI IN, et al., 2018).

O quadro é mais comum no pós-parto, sendo também mais seguro seu tratamento nesse período, já que na gestação algumas medicações para o tratamento da doença são contraindicadas, como os IECA, sacubitril/valsartana, Ivabradina, novos anticoagulantes e Espironolactona. Ainda, deve-se ajustar as medicações após o parto, se a puérpera estiver amamentando, onde são contraindicados os BRA, sacubitril/valsartana, Ivabradina e novos anticoagulantes. A terapêutica principal deve ser mantida de 12 a 24 meses, com as seguintes drogas: betabloqueadores, IECA/BRA e Espironolactona (DAVIS M, et al., 2019).

Para essa gestante, foi optado por realização de cesárea com laqueadura tubária. Sabe-se que a via de parto é decidida de acordo com a gravidade do caso. Em pacientes estáveis, o parto vaginal é indicado, pois complicações como embolia pulmonar, endometrite ou edema são mais comuns em parto cesáreo. Para isso, é necessária monitorização hemodinâmica de mãe/feto e controle da dor para evitar sobrecarga cardíaca materna. Se instáveis, a melhor opção é a cesariana (BORDIGNON S, 2007).

A anestesia realizada para o parto cesárea poderia levar a piora do quadro, visto que complicações cardiovasculares estão descritas neste ato. Além disso, a própria cirurgia causa aumento da resposta inflamatória, a qual poderia desencadear desestabilização do quadro prévio da paciente, com piora da congestão pulmonar e edema. O que não ocorreu, visto que após a resolução da gestação, paciente permaneceu estável hemodinamicamente e pode ser tratada com medicações mais efetivas para o quadro, como o IECA já citado (SANTOS SM, et al., 2012).

O planejamento familiar é essencial nesses casos, pois existe o risco de novas descompensações em gestações futuras. A contracepção então, deve ser discutida, podendo considerar métodos de longa ação, como dispositivos intrauterinos (DIU) ou até mesmo métodos irreversíveis, se o comprometimento da função 
ventricular for grave. O método de escolha para esta paciente foi a laqueadura tubária, devido à gravidade do quadro apresentado. A mesma foi orientada sobre os riscos de uma nova gestação e manifestou o desejo sobre tal ato (BORDIGNON S, 2007).

Segundo a Lei do planejamento familiar, Lei no 9.263/96, a esterilização cirúrgica feminina é permitida apenas quando a mulher apresenta mais que 25 anos ou mais que 2 filhos vivos, a realização do ato cirúrgico e o manifesto da paciente necessita de um intervalo de 60 dias. Entretanto, isso é exceção se houver doença materna grave, pelo risco de um novo ato cirúrgico e descompensação cardiovascular (CONSELHO REGIONAL DE MEDICINA DO ESTADO DO PARANÁ, 2018).

Em relação ao prognostico da doença, a função ventricular e a área cardíaca se encontram normais em $50 \%$ dos casos após um período de 6 a 12 meses, podemos verificar isso na paciente descrita, visto que inicialmente a FE<29\% e progrediu para FE 52\% após alguns meses (FILHO FMA e SILVA TT, 1999).

Em suma, este relato apresenta uma gestante jovem e previamente hígida que evoluiu para um quadro grave com inúmeras complicações maternas e fetais que se não fosse identificado a tempo, poderia levar a evolução clinica desfavorável. Portanto, apesar da Miocardiopatia Periparto ser uma patologia rara, seu diagnóstico precoce é imprescindível para reduzir as taxas de morbimortalidade no país e para isso, é necessário estar atentos aos sinais e sintomas cardiorrespiratórios de todas as gestantes no acompanhamento do pré-natal.

\section{REFERÊNCIAS}

1. AVILA W, et al. Gravidez em Portadoras de Cardiomiopatia Periparto. Arquivos Brasileiros de Cardiologia, 2002; 79(5).

2. BORDIGNON S. Cardiomiopatia periparto: contra-indicação para subseqüentes gravidezes. Revista da Sociedade de Cardiologia do Rio Grande do Sul, 2007; 11.

3. CÈNAC A, et al. A low plasma selenium is a risk factor for peripartum cardiomyopathy. Internation Journal of Cardiology, 1992; 36.

4. CONSELHO REGIONAL DE MEDICINA DO ESTADO DO PARANÁ. Cirurgia de laqueadura tubária concomitante ao parto, 2018.

5. DAVIS M, et al. Peripartum Cardiomyopathy. Journal of the American College of Cardiology, 2019; 75(2).

6. DEMAKIS J, RAHIMTOOLA S. Peripartum cardiomyopathy. Circulation, 1971; 44.

7. FILHO FMA, SILVA TT. O comportamento das gestações subsequentes na cardiopatia periparto. Arquivos Brasileiros de Cardiologia, 1999;73(1): 47-52.

8. HOMANS DC. Peripartum Cardiomyopathy. The New England Journal of Medicine, 1985; 312.

9. LEONARD R, et al. Peripartum cardiomyopathy. Journal of Emergency Medicine, 1992; 10.

10. MOIOLI M, et al. Peripartum cardiomyopathy. Archivesof Gynecology Obstetrics, 2010; 183-8.

11. NAPOLI IN, et al. Miocardiopatia periparto em hospital universitário do sul de Minas Gerais: relato de caso. Revista Médicade Minas Gerais, 2018; 28(8).

12. OLIVEIRA FM, et al. Cardiomiopatia periparto. Revista Médica de Minas Gerais, 2012; 22(5).

13. PATTA C, NUNES R. Miocardiopatia Periparto. Arquivos Catarinenses de Medicina, 2012; 41 (3).

14. PEARSON G, et al. Peripartum cardiomyopathy. JAMA, 2000; 283:1183.

15. PINTO C, et al. Miocardiopatia Periparto. Acta Médicade Portugal, 2007; 20.

16. PYATT J, DUBEY G. Peripartum cardiomyopathy: current understanding,comprehensive management review and new developments. Postgraduate Medical Journal, 2011; 87(1023).

17. RESENDE B, et al. Miocardiopatia Periparto. Revista Médicade Minas Gerais, 2009; 19(4).

18. RICKENBACHER $P$, et al. Long-term outcome after heart transplantation for peripartum cardiomyopathy. American Heart Journal, $1994 ; 127$.

19. ROMBALDI A, et al. Cardiomiopatia Periparto. Sociedade de Cardiologia do Rio Grande do Sul, 2005;(5).

20. SANTOS SM, et al. Miocardiopatia periparto após cesariana:estudo de casos. Brasília Médica, 2012; 49(4).

21. SIMÕES M, et al. Miocardiopatia Periparto. Acta Obstétrica de Ginecologia Portuguesa, 2015; 9(4).

22. SLIWA K, et al. Peripartum Cardiomyopathy. Lancet, 2006; 368(9536).

23. SLIWA K, et al. Current state of knowledge on aetiology,diagnosis, management, and therapy of peripartum cardiomyopathy. European Journal of Heart Fail, 2010: 767-778.

24. TEDOLDI C. Miocardiopatia periparto. 2009. Diretriz da Sociedade Brasileira de Cardiologia para Gravidez na Mulher Portadora de Cardiopatia. Rio de Janerio: da Sociedade Brasileira de Cardiologia. 